\title{
Ethanol-induced intestinal miRNAs in liver injury and early development of liver cancer
}

\author{
Je-Hyun YOON* \\ Department of Biochemistry and Molecular Biology, Medical University of South Carolina, Charleston, SC, USA
}

Introduction: While binge alcohol-induced gut leakage has been studied extensively in the context of ROS-mediated signaling, it was recently revealed that post-transcriptional regulation plays an essential role as well. Ethanol-inducible CYP2E1 is a key enzyme in ethanol metabolism, and promotes alcohol-induced hepatic steatosis and inflammatory liver disease at least in part by mediating changes in intestinal permeability. For instance, gut leakage and elevated intestinal permeability to endotoxins have been shown to be regulated by enhancing CYP2E1 mRNA and CYP2E1 protein levels. Although it is understood that ethanol promotes CYP2E1 induction and activation, the mechanisms by which CYP2E1 expression is regulated in the context of intestinal damage remain poorly defined. Specific miRNAs have been shown to repress the expression of CYP2E1, supporting our hypothesis that these miRNAs contribute to ethanol-induced intestinal injury and early development of liver cancer.

Methods: We utilized in vitro cell model and in vivo mouse model to study intestinal and hepatic cell death and growth after modulation of specific miRNA levels directly or indirectly by depletion or overexpression of microRNA-binding proteins.

Results: We made two key observations that 1) the RNA-binding protein AUF1 binds mature miRNAs, including CYP2E1-targeting miRNAs, and this binding modulates the degradation of corresponding target mRNAs; 2) the Serine/Threonine kinase MST1 mediates oxidative stress-induced phosphorylation of RNA-binding proteins such as AUF1. This finding suggests that ROS-mediated signaling modulates AUF1/miRNA interaction through MST1-mediated phosphorylation for developing liver cancer.

Conclusions: Thus, our study demonstrated the critical functions of AUF1 phosphorylation by MST1 in intestine injury and liver cancer development. 